\title{
The Primary Care PTSD Screen for DSM-5 (PC-PTSD-5): Development and Evaluation Within a Veteran Primary Care Sample
}

\author{
Annabel Prins, $P h D^{1,2}$, Michelle J. Bovin, $P h D^{3,4}$, Derek J. Smolenski, PhD, MPH ${ }^{5}$, Brian P. Marx, $P h D^{3,4}$, \\ Rachel Kimerling, PhD ${ }^{7,6}$, Michael A. Jenkins-Guarnieri, $P h D^{7}$, Danny G. Kaloupek, PhD ${ }^{3,4}$, \\ Paula P. Schnurr, PhD ${ }^{8,9}$, Anica Pless Kaiser, PhD ${ }^{3,4}$, Yani E. Leyva, PhD ${ }^{10}$, and Quyen Q. Tiet, \\ $P h D^{1,6,17,12}$
}

\begin{abstract}
'National Center for PTSD, VA Palo Alto Health Care System, Menlo Park, CA, USA; '²Department of Psychology, San Jose State University, San Jose, CA, USA; ${ }^{3}$ National Center for PTSD, VA Boston Healthcare System, Boston, MA, USA; ${ }^{4}$ Boston University School of Medicine, Boston, MA, USA; ${ }^{5}$ National Center for Telehealth and Technology, Joint Base Lewis-McChord, WA, USA; ${ }^{6}$ Center for Innovation to Implementation, VA Palo Alto

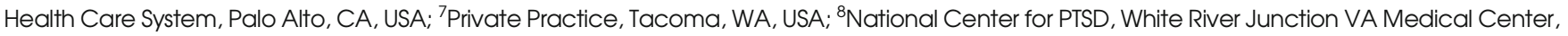
White River Junction, VT, USA; ${ }^{9}$ Geisel School of Medicine at Dartmouth, Hanover, NH, USA; ${ }^{10}$ Research Division, San Francisco VA Medical Center, San Francisco, CA, USA; "'Department of Psychiatry and Behavioral Sciences, Stanford University School of Medicine, Stanford, CA, USA;

${ }^{12}$ California School of Professional Psychology, Alliant International University, San Francisco, CA, USA.
\end{abstract}

BACKGROUND: Posttraumatic Stress Disorder (PTSD) is associated with increased health care utilization, medical morbidity, and tobacco and alcohol use. Consequently, screening for PTSD has become increasingly common in primary care clinics, especially in Veteran healthcare settings where trauma exposure among patients is common. OBJECTIVE: The objective of this study was to revise the Primary Care PTSD screen (PC-PTSD) to reflect the new Diagnostic and Statistical Manual of Mental Disorders (DSM-5) criteria for PTSD (PC-PTSD-5) and to examine both the diagnostic accuracy and the patient acceptability of the revised measure.

DESIGN: We compared the PC-PTSD-5 results with those from a brief psychiatric interview for PTSD. Participants also rated screening preferences and acceptability of the PC-PTSD-5.

PARTICIPANTS: A convenience sample of 398 Veterans participated in the study (response rate $=41 \%$ ). Most of the participants were male, in their 60s, and the majority identified as non-Hispanic White.

MEASURES: The PC-PTSD-5 was used as the screening measure, a modified version of the PTSD module of the MINI-International Neuropsychiatric Interview was used to diagnose DSM-5 PTSD, and five brief survey items were used to assess acceptability and preferences.

KEY RESULTS: The PC-PTSD-5 demonstrated excellent diagnostic accuracy (AUC $=0.941 ; 95 \%$ C.I.: 0.9120.969). Whereas a cut score of 3 maximized sensitivity $(\kappa[1])=0.93 ; \mathrm{SE}=.041 ; 95 \%$ C.I.: 0.849-1.00), a cut score of 4 maximized efficiency $(\kappa[0.5]=0.63 ; \mathrm{SE}=0.052 ; 95 \%$ C.I.: 0.527-0.731), and a cut score of 5 maximized specificity $(\kappa[0]=0.70 ; \mathrm{SE}=0.077 ; 95 \%$ C.I.: 0.550-0.853). Patients found the screen acceptable and indicated a preference for administration by their primary care providers as opposed to by other providers or via self-report.

Received November 17, 2015

Revised March 18, 2016

Accepted March 29, 2016

Published online May 11, 2016
CONCLUSIONS: The PC-PTSD-5 demonstrated strong preliminary results for diagnostic accuracy, and was broadly acceptable to patients.

KEY WORDS: PTSD; screening; DSM-5; primary health care.

J Gen Intern Med 31(10): 1206-11

DOI: $10.1007 / \mathrm{s} 11606-016-3703-5$

(C) Society of General Internal Medicine 2016

$\mathrm{T}$ he prevalence of posttraumatic stress disorder (PTSD) in Veteran primary care samples is two to three times higher than PTSD prevalence in the general population (10-20\% versus $6-7 \%)^{1-3}$ and is similar to the prevalence of depression and generalized anxiety disorder in primary care settings ( $18.9 \%$ and $14.8 \%$, respectively), ${ }^{4,5}$ PTSD is consistently associated with increased health care utilization, medical morbidity, and health-compromising behaviors such as tobacco and alcohol abuse. ${ }^{6}$ The high prevalence and negative health effects of PTSD have led to increased screening efforts for the disorder in Department of Veterans Affairs (VA) and Department of Defense (DoD) clinics. ${ }^{7,8}$

The Primary Care PTSD Screen (PC-PTSD) ${ }^{9}$ is a four-item measure that reflects the Diagnostic and Statistical Manual of Mental Disorders, fourth edition (DSM-IV) PTSD diagnostic criteria. ${ }^{10}$ Using a score of three as the screening threshold, ${ }^{9}$ the PC-PTSD demonstrates better operating characteristics than other screening measures, including the Single-Item PTSD Screen (SIPS), the General Health Questionnaire (GHQ-12), and the Startle, Physiological arousal, Anxiety, Numbness (SPAN) questionnaire., ${ }^{211-13}$ Further, Freedy and his colleagues found that the PC-PTSD performed similarly to the PTSD Checklist - Civilian version (PCL-C) ${ }^{2}$, a wellestablished longer measure of PTSD that assesses the full range of symptoms. ${ }^{14,15}$ Because the PC-PTSD has demonstrated excellent diagnostic accuracy and clinical utility with 
various groups of primary care patients, ${ }^{2,9,16,17}$ it is mandated for use in VA and DoD settings. ${ }^{18,19}$

In 2013, the fifth edition of the Diagnostic and Statistical Manual (DSM-5) ${ }^{20}$ introduced significant revisions to the PTSD diagnosis based on research suggesting that these changes better reflect the PTSD latent construct. Consistent with widespread recognition that fear and anxiety represent only one phenotypic expression of the disorder, PTSD is now placed in a new Trauma- and Stressor-Related Disorders category. ${ }^{21}$ Additional changes to the diagnostic criteria are threefold. First, the trauma exposure criterion no longer requires the individual to experience fear, helplessness, or horror, due to research suggesting that this did not affect PTSD prevalence. ${ }^{22}$ Second, the wording of several core symptoms was revised, and three additional symptoms reflecting distorted blame, persistent negative affect (sadness, anger, guilt), and risk-taking behaviors were added to reflect research highlighting the importance of these symptoms. ${ }^{22}$ Third, based on factor analytic studies, PTSD symptoms are now grouped into four clusters instead of three. ${ }^{23}$

As a result of these revisions to the diagnostic criteria, we made two major changes to the PC-PTSD. Specifically, we altered the trauma-defining stem and added one additional item that assesses trauma-distorted blame and guilt. We did not include risk-taking in our revision because this item has not demonstrated the same discriminative validity and strong factor loading as the blame and guilt items. ${ }^{24}$

In this paper, we report results of diagnostic accuracy analyses of the PC-PTSD-5 in a Veteran primary care sample. We also describe patient preferences for administration mode and general acceptability of the measure. We expected the PCPTSD-5 to have good diagnostic accuracy and to be well accepted by primary care patients.

\section{METHOD}

PC-PTSD-5 Development. Consistent with standards for measure development, ${ }^{25}$ existing DSM-5 measures of trauma exposure and PTSD were reviewed for item consideration. Candidate items were then evaluated by five to seven PTSD measurement experts for clarity of wording and consistency with DSM-5 criteria. Items were revised until consensus was reached, and a dichotomous (yes/no) response format was chosen instead of a Likert scale to facilitate administration and scoring. The revised trauma exposure stem was designed such that individuals who do not report trauma exposure do not answer subsequent questions about PTSD symptoms; this prevents unnecessary administration of the remaining PC-PTSD-5 items and makes the screen even shorter for the $40-50 \%$ of patients who are not trauma exposed. ${ }^{1}$ We conducted a pilot study to evaluate the diagnostic accuracy of the PCPTSD and the PC-PTSD-Revised Stem. ${ }^{26}$ There were no significant differences in the Area Under the Curve (AUC) between the two screens; both screens had excellent diagnostic accuracy $(A U C>0.92)$ and clinical significance ${ }^{27}$ $(\kappa \geq 0.75)$, and both screens had an optimally sensitive cutoff score of 3 .

The fifth item was added to assess the new symptom cluster of negative alterations in mood and cognitions, particularly guilt and blame based on research highlighting their centrality to the diagnosis. ${ }^{22}$ Revisions of candidate items focused on wording that was easy to understand and referenced to traumatic event(s). The resulting screen has a Flesch-Kincaid reading level of grade 6.5. To reflect our DSM-5 focus and the addition of a fifth item, we renamed the screen PC-PTSD-5.

Procedure. The current data were collected as part of the Drug Screen for Primary Care Patients Study. ${ }^{28}$ The primary aim of this larger project was to develop and validate a brief drug screening instrument for VA primary care settings. The Drug Screen study, including all aspects of our embedded study, was approved by the local Institutional Review Board.

Data collection for the current study occurred between March and December 2013. Project staff recruited patients in the waiting areas of two VA primary care clinics. All patients currently receiving primary care at one of the clinics were eligible to participate. Exclusion criteria were based on inability to provide informed consent. Participants were interviewed by a trained research assistant, and received $\$ 30$ in the form of a VA voucher or commercial gift card for their participation.

Participants. Of the 962 consecutive Veterans approached for participation, 398 (41 \%) participated. Participants ranged in age from 24 to 89 years $(M=63.3$ years, $S D=12.4$ years). Participants were predominantly male (96.0 \%) and non-Hispanic White (57.4 \%), with $12.1 \%$ identifying as non-Hispanic African American, $9.6 \%$ as Hispanic of any race, $5.5 \%$ as Asian, $0.5 \%$ as Native American, and $14.9 \%$ as Other.

\section{Measures}

PC-PTSD-5. PC-PTSD-5 content is presented in Text Box 1 and is identical to the PC-PTSD, with the exception of the revised stem and the addition of a fifth item which captures trauma-specific guilt and blame. Previous research with the PC-PTSD has shown good test-retest reliability $(r=0.83)$ and predictive validity against the Clinician Administered PTSD Scale (CAPS; $r=0.83$ ). ${ }^{9}$ In this study, the PC-PTSD-5 was administered verbally by a research assistant. 
Text Box 1. The Primary Care PTSD Screen for DSM-5 (PC-PTSD-5)

Sometimes things happen to people that are unusually or especially frightening, horrible, or traumatic. For example:

- a serious accident or fire

- a physical or sexual assault or abuse

- an earthquake or flood

- a war

- seeing someone be killed or seriously injured

- having a loved one die through homicide or suicide

Have you ever experienced this kind of event?

If 'No,' screen total $=0$; if 'Yes,' continue with screening

In the past month, have you...

1. had nightmares about the event(s) or thought about the event(s) when you did not want to?

2. tried hard not to think about the event(s) or went out of your way to avoid situations that reminded you of the event(s)?

3. been constantly on guard, watchful, or easily startled?

4. felt numb or detached from people, activities, or your surroundings?

5. felt guilty or unable to stop blaming yourself or others for the events(s) or any problems the event(s) may have caused?

Note. All new items are italicized here for emphasis; respondents were asked to answer "yes" or "no" to all items.

\section{MINI-International Neuropsychiatric Interview (MINI).} The $\mathrm{MINI}^{29}$ is a short, semi-structured, diagnostic interview designed to be administered by trained lay interviewers. It has good concordance with the Structured Clinical Interview for $\mathrm{DSM}^{30}$ (SCID) and the Composite International Diagnostic Interview. ${ }^{31}$ The MINI is compatible with both the International Classifications of Diseases-10 and the DSM-IV. There is good concordance between the PTSD module of the MINI and the PTSD section of the SCID. ${ }^{29,32}$

At the time of the study, no clinical interviews for DSM-5 PTSD had completed validation studies. For this reason, a modified version of the DSM-IV PTSD module of the MINI was used to assess DSM-5 criteria. These alterations included a trauma probe that read:

Have you ever experienced or witnessed or had to deal with an extremely traumatic event that included actual or threatened death or serious injury to you or someone else? Examples of traumatic events include: serious accidents, sexual or physical assault, a terrorist attack, being held hostage, kidnapping, fire, discovering a body, sudden death of someone close to you, war, or natural disaster.

Have you ever learned about a violent or accidental death, or threatened death, that happened to a close relative or close friend?

Symptoms also were added or modified to be consistent with DSM-5. This involved no changes to the intrusion and avoidance criteria, the addition of two new items to reflect alterations in mood and cognition (e.g., guilt and blame), and the addition of one arousal item to capture reckless behavior. A PTSD diagnosis was established if the DSM-5 symptom criteria algorithm was met and symptoms caused significant distress and/or impairment. The modified MINI was administered by trained research assistants who audiotaped their interviews. Randomly selected interviews were reviewed for $11.1 \%$ of the sample by two independent raters who obtained perfect inter-rater reliability at the diagnostic level and excellent inter-rater reliability $(\kappa>0.95)$ at the item level. ${ }^{28}$
Acceptability Questionnaire. A five-item questionnaire was used to assess respondents' experience with the PC-PTSD-5 for a subset of participants $(n=253)$. The first question assessed the degree to which the screen was easy to understand using a five-point rating scale from $1=$ very easy to understand to $5=$ very difficult to understand. The second question asked how comfortable participants would be completing the PC-PTSD-5 during a primary care visit on a scale from $1=$ very comfortable to $5=$ very uncomfortable. The final three questions asked about preferred mode of PC-PTSD-5 administration at a primary care encounter. Options included completing the screen on their own, administration by a primary care doctor, and administration by a nurse or other provider. Ratings were recorded on a scale from $1=$ very comfortable to $5=$ very uncomfortable.

Data Analysis. Diagnostic accuracy of the PC-PTSD-5 was examined with weighted $\mathrm{K}$ coefficients as measures of test quality, including quality of sensitivity $(\kappa[1])$, specificity $(\kappa[0])$, and efficiency $(\kappa[0.5])$. Unlike commonly reported measures of test performance (e.g., sensitivity, specificity, and efficiency), weighted $\mathrm{k}$ coefficients are calibrated for chance agreement between test and diagnosis. ${ }^{33}$ Guidelines developed for judging levels of clinical significance suggest that $k \leq 0.40$ is poor, $\geq 0.41$ and $<60$ is fair, $\geq 0.60$ and $<0.75$ is good, and $\geq 0.75$ is excellent. ${ }^{27}$

We identified the optimally sensitive cutoff (i.e., the PC-PTSD-5 cutoff with the highest $\mathrm{k}[1]$ ) that also had a specificity $\geq 0.80 .^{34,35}$ This insured that our optimally sensitive cutoff (ideal for screening ${ }^{33}$ ) would not capture too many false positives. Using DAG_STAT ${ }^{36}$, we calculated measures of test performance (sensitivity, specificity, efficiency, positive and negative predictive values, and positive and negative likelihood ratios) and test quality (kappa coefficients representing quality of sensitivity, specificity, and efficiency) for all possible PC-PTSD-5 scores (1-5). We also calculated means for the two items assessing ease of understanding and comfort with completing the screen, and conducted $t$-tests to determine the preferred mode of administration. 
Table 1. Estimates of Classification Quality of the PC-PTSD-5 for the DSM-5 Diagnosis of PTSD

\begin{tabular}{|c|c|c|c|c|c|c|c|c|c|c|}
\hline PC-PTSD-5 Score & 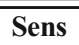 & Spec & Eff & $\overline{\text { PPV }}$ & NPV & $\overline{\text { LR+ }}$ & LR- & $\overline{c k(0)}$ & $\overline{~ K(.5)}$ & K(1) \\
\hline$\overline{0}$ & - & - & - & - & - & - & - & - & - & - \\
\hline 1 & 0.99 & 0.67 & 0.72 & 0.33 & 1.00 & 2.99 & 0.03 & 0.22 & 0.36 & 0.97 \\
\hline 2 & 0.98 & 0.78 & 0.81 & 0.42 & 1.00 & 4.41 & 0.02 & 0.33 & 0.49 & 0.97 \\
\hline 3 & 0.95 & 0.85 & 0.86 & 0.51 & 0.99 & 6.33 & 0.06 & 0.43 & 0.59 & 0.93 \\
\hline 4 & 0.83 & 0.91 & 0.90 & 0.60 & 0.97 & 8.79 & 0.19 & 0.53 & 0.63 & 0.78 \\
\hline 5 & 0.56 & 0.97 & 0.91 & 0.74 & 0.93 & 17.40 & 0.45 & 0.70 & 0.59 & 0.51 \\
\hline
\end{tabular}

Italics indicates optimally sensitive cutoff score. Sens sensitivity; Spec specificity; Eff efficiency; PPV positive predictive value; NPV negative predictive value; $L R+$ positive likelihood ratio; LR- negative likelihood ratio; $\kappa(0)$ quality of specificity; $\kappa(0.5)$ quality of efficiency; $\kappa(1)$ quality of sensitivity

\section{RESULTS}

Preliminary Analyses. Per the MINI, $14.3 \%$ of the sample was diagnosed with PTSD. There were no significant differences between participants who were administered the acceptability questionnaire and those who were not on gender, race, marital status, education, annual income or PTSD status (all $\chi^{2}<7.44$; all $p s>0.05$ ), although participants who were administered the acceptability questionnaire were somewhat younger $(M=62.34, S D=12.33$ vs. $M=65.02, S D=12.37$; $t(395)=2.09, p=0.03)$.

Diagnostic Accuracy. The AUC for the PC-PTSD-5 was 0.941 (95\% C.I.: 0.912-0.969). The optimally sensitive score for the PC-PTSD-5 was 3. This cut score produced the highest $\mathrm{k}(1)$ score $(\kappa[1]=0.93 ; \mathrm{SE}=.041 ; 95 \%$ C.I.: 0.849-1.00), while still demonstrating acceptable specificity $(\geq 0.80)$. The optimally efficient cut score $(\kappa[0.5])$ was $4(\kappa[.5]=0.63$; $\mathrm{SE}=0.052$; $95 \%$ C.I.: $0.527-0.731)$. A cut score of 5 maximized specificity $(\kappa[0]=0.70 ; \mathrm{SE}=0.077 ; 95 \%$ C.I.: 0.550-0.853; see Table 1).

Acceptability Analyses. Participants indicated that the screen was easy to understand $(M=1.73 ; S D=0.80)$ and that they would feel comfortable completing it during a primary care visit $(M=1.75 ; S D=0.92)$. Participants preferred screening by their primary care provider over a nurse or other provider $[t(252)=-5.539, p<0.001]$ or by self-administration $[t(252)$ $=5.184, p<0.001$; see Table 2].

\section{DISCUSSION}

This is the first study to provide evidence of the PC-PTSD-5's utility in screening for DSM-5 PTSD. Diagnostic utility characteristics of the PC-PTSD-5 were excellent, and are similar to those reported for the PHQ-9, a frequently used primary care screen for depression. ${ }^{37}$ This study also provided evidence that the PC-PTSD-5 is easy to understand and appropriate for primary care settings. Overall, the PC-PTSD-5 appears to be a diagnostically accurate screen that is content and face valid relative to DSM-5 criteria.

We found that the optimally sensitive cutoff score was 3 . Optimizing sensitivity minimizes false negative screen results, which is desirable because the purpose of primary care screening is to facilitate early detection and treatment of PTSD cases that would otherwise go unrecognized. With a cutoff of 3, we identified $94.8 \%$ of participants who were diagnosed with PTSD using the MINI. In contrast, a cutoff of 4 identified $82.6 \%$ of participants diagnosed with PTSD, and a cutoff of 5 identified only $56.2 \%$ of PTSD participants. However, minimizing false negative screens potentially inflates the number of false positive screens. For example, using a cutoff of 3 , $26.4 \%$ of our sample would be identified by the PC-PTSD-5 as having PTSD, which is substantially more than the number of participants identified by the MINI as having PTSD (14.3\%). Cutoffs of 4 (19.8 vs. $14.3 \%$ participants with PTSD) and 5 (10.8 vs. $14.3 \%$ participants with PTSD) would reduce the number of false positives.

Table 2. Patient Perceptions of PC-PTSD-5 Measure Administration

\begin{tabular}{|c|c|c|c|c|c|}
\hline Variables & $\begin{array}{l}\text { Very Easy/ } \\
\text { Comfortable } \\
n(\%)\end{array}$ & $\begin{array}{l}\text { Easy/ } \\
\text { Comfortable } \\
n(\%)\end{array}$ & $\begin{array}{l}\text { Not Sure } \\
n(\%)\end{array}$ & $\begin{array}{l}\text { Difficult/ } \\
\text { Uncomfortable } \\
n(\%)\end{array}$ & $\begin{array}{l}\text { Very Difficult/ } \\
\text { Uncomfortable } \\
n(\%)\end{array}$ \\
\hline $\begin{array}{l}\text { Understand the question } \\
\text { Answer the question at a PC visit } \\
\text { Fill out a paper/e-form on own } \\
\text { Asked by PC doctor } \\
\text { Asked by a nurse/other } \\
\text { providers }\end{array}$ & $\begin{array}{l}111(43.9) \\
117(46.2) \\
79(31.2) \\
97(38.3) \\
79(31.2)\end{array}$ & $\begin{array}{l}114(45.1) \\
104(41.1) \\
113(44.7) \\
127(50.2) \\
114(45.1)\end{array}$ & $\begin{array}{l}14(5.5) \\
14(5.5) \\
33(13.0) \\
13(5.1) \\
30(11.9)\end{array}$ & $\begin{array}{l}14(5.5) \\
13(5.1) \\
18(7.1) \\
11(4.3) \\
19(7.5)\end{array}$ & $\begin{array}{l}0(0.0) \\
5(2.0) \\
10(4.0) \\
5(2.0) \\
11(4.3)\end{array}$ \\
\hline
\end{tabular}


Screening for different purposes, populations, or settings will require careful evaluation of the most useful cut score for the PC-PTSD-5. In some cases, it may be more important to balance the ability to detect PTSD with the need to conserve the resources required to address positive screens among patients with absent or subthreshold diagnoses. In settings where the prevalence of PTSD is likely to be substantially higher than primary care (e.g., mental health clinics), a more conservative approach may be desired, utilizing either the optimally efficient or optimally specific cutoff scores. Thus, for any screening program, consideration of the benefits of case finding and the cost of false positives, as well as the base rate of PTSD, is necessary.

The PC-PTSD-5 appears to be well tolerated by primary care patients. A large majority of participants found the PCPTSD-5 easy to understand and would feel comfortable completing it during a primary care visit. Our results also indicated that participants would generally prefer to have the screen administered by a primary care doctor, rather than through self-administration or by a nurse or other medical assistant. This type of information helps to ensure that screening programs that use the PC-PTSD-5 are patient-centered in terms of implementation. To enhance utility, it may be valuable to learn more about whether the screen is equally acceptable among all Veteran subgroups. Further, additional research is needed to determine how to effectively achieve patient-centered administration within busy primary care clinics, in which primary care doctors may not always be able to administer the instrument.

It is worth noting that, although the diagnostic accuracy statistics generated by the original PC-PTSD, the PC-PTSDRevised Stem, and the PC-PTSD-5 are very similar, this does not guarantee that each screen is identifying the same individuals as having probable PTSD. Indeed, in a study that compared the DSM-IV PTSD Checklist (PCL-S) to the DSM-5 version of the measure (PCL-5), Hoge and his colleagues found that whereas PTSD prevalence according to the two instruments was nearly identical (13 versus $12 \%$ ), a high percentage of participants who met criteria by one definition did not meet the other (i.e., $45 \%$ of participants meeting either criteria had discordant results). ${ }^{38}$ Further, similar to our findings, Hoge et al. found that the diagnostic utility of the PCL was not changed despite these disparities regarding which participants were categorized as having PTSD. ${ }^{38}$ Because DSM-5 represents the most up-to-date knowledge of the PTSD construct, it is essential that we have a screener that can identify patients with probable PTSD based on this definition. Our results suggest that the PC-PTSD-5, which was designed to reflect the DSM-5 PTSD definition, does that without compromising the excellent diagnostic utility of its predecessor.

The reliance on a convenience sample that was predominantly male and older may limit the generalizability of the findings. Other aspects of the study, such as potential participant self-selection, may also limit study generalizability. Specifically, Veterans with more severe PTSD (particularly avoidance) symptoms may have been more likely to refuse to participate in this study. However, since the study was not advertised as a PTSD study, recruitment may not have triggered PTSD-related responses such as avoidance, increasing the likelihood that these individuals were included in our sample. Further, because the rate of PTSD in our sample was comparable to rates of PTSD in other primary care samples, ${ }^{3,39,40}$ we are cautiously optimistic that our results are generalizable. It is also possible that many of these participants had already been screened for PTSD and/or given a PTSD diagnosis prior to the study. Although in these cases the diagnosis was likely based on DSM-IV version, it could still potentially affect diagnostic accuracy and acceptability outcomes. Replication of the study with a sample that is naïve to their PTSD status is needed.

Another limitation is that we did not use a validated gold standard DSM-5 PTSD interview as the diagnostic criterion. Although the DSM-IV PTSD module of the MINI has been used in other validation studies ${ }^{41}$ and our modifications to the MINI were consistent with the DSM-5 PTSD diagnosis, future studies will need to use a validated clinician administered diagnostic measure like the CAPS- $5 .{ }^{42}$ However, our results are an important first step that allows for the implementation of the PC-PTSD-5 in primary care clinics and which, we hope, will encourage additional research exploring whether the cutoffs reported here are appropriate for all settings and populations. Finally, the results of our pilot ${ }^{26}$ and current study do not take comorbidity or other spectrum effects (i.e., variation among the groups with and without PTSD) into consideration. ${ }^{43}$ It is possible that individuals with certain characteristics (e.g., demographic features or comorbid psychopathology) require different cutoff scores. Future research is needed to examine this possibility.

Overall, the findings indicate that the PC-PTSD-5 possesses strong diagnostic accuracy. The revised screening tool represents an advance beyond the PC-PTSD by reflecting the field's most up-to-date knowledge of the PTSD construct, while still maintaining the strengths of the original screener. Additional research is needed to confirm the cutoff scores identified here, but our findings suggest that the new screener is acceptable to primary care patients and that it can be used to effectively identify individuals with probable PTSD. 
Acknowledgments: We thank the VA patients who participated in the study as well as the research support provided by Frank W. Weathers, Ph.D., Brandy N. Smith, B.A., Alexandra Klein, B. A., and Aaron Harwell, B. A. Data collection for this project was funded by a VA Health Services Research and Development grant awarded to Dr. Tiet (IIR10-347). An earlier version of this work was presented at the International Society for Traumatic Stress Studies (ISTSS), Miami, FL, November 6-8, 2014. The views expressed in the article are those of the authors and do not necessarily represent the views of the Department of Veterans Affairs or the United States Government.

Corresponding Author: Annabel Prins, PhD; Department of Psychology San Jose State University, One Washington Square, San Jose, CA 95192, USA (e-mail: Annabel.Prins@sjsu.edu).

\section{Compliance with Ethical Standards}

Conflict of Interest: The authors declare no conflicts of interest.

\section{REFERENCES}

1. Breslau N. The epidemiology of trauma, PTSD, and other posttrauma disorders. Trauma Violence Abuse. 2009; 10(3): 198-210.

2. Freedy JR, Steenkamp MM, Magruder KM, et al. Post-traumatic stress disorder screening test performance in civilian primary care. Fam Pract. 2010;27(6):615-24

3. Magruder KM, Frueh BC, Knapp RG, et al. Prevalence of posttraumatic stress disorder in veterans affairs primary care clinics. Gen Hosp Psychiatry. 2005;27(3): 169-79.

4. Milanak ME, Gros DF, Magruder KM, Brawman-Mintzer O, Frueh BC. Prevalence and features of generalized anxiety disorder in department of veteran affairs primary care settings. Psychiatry Res. 2013;209(2):173-9.

5. Olfson M, Shea S, Feder A, et al. Prevalence of anxiety, depression, and substance use disorders in an urban general medicine practice. Arch Fam Med. 2000;9(9):876-83.

6. Schnurr PP. Understanding pathways from traumatic exposure to physical health. Evid Based Treat Trauma-Relat Psychol Disord: Springer; 2015:87-103.

7. Belsher BE, Curry J, McCutchan P, et al. Implementation of a collaborative care initiative for PTSD and depression in the army primary care system. Soc Work Ment Health. 2014;12(5-6):500-22.

8. Tsan JY, Zeber JE, Stock EM, Sun F, Copeland LA. Primary care-mental health integration and treatment retention among Iraq and Afghanistan war veterans. Psychol Serv. 2012;9(4):336-48.

9. Prins, A, Ouimette, $\mathbf{P}$, Kimerling, $\mathbf{R}$, et al . The primary care PTSD screen (PC-PTSD): development and operating characteristics. Primary Care Psychiatry. 2003;9(1):9-14

10. American Psychiatric Association. Diagnostic and statistical manual of mental disorders-4th edition (DSM-IV). Arlington: APA; 1994.

11. Gore KL, Engel CC, Freed MC, Liu X, Armstrong DW. Test of a singleitem posttraumatic stress disorder screener in a military primary care setting. Gen Hosp Psychiatry. 2008;30(5):391-7.

12. Ouimette P, Wade M, Prins A, Schohn M. Identifying PTSD in primary care: comparison of the primary care-PTSD screen (PC-PTSD) and the General Health Questionnaire-12 (GHQ). J Anxiety Disord. 2008:22(2):337-43.

13. Spoont MR, Williams JW, Kehle-Forbes S, Nieuwsma JA, Mann-Wrobel MC, Gross R. Does this patient have posttraumatic stress disorder? Rational clinical examination systematic review. JAMA. 2015;314(5):501-10.

14. Blanchard EB, Jones-Alexander J, Buckley TC, Forneris CA. Psychometric properties of the PTSD Checklist (PCL). Behav Res Ther. 1996;34(8):669-73.

15. Weathers FW, Litz BT, Herman DS, Huska JA, Keane TM. The PTSD Checklist (PCL): reliability, validity, and diagnostic utility. Paper presented at the annual meeting of the International Society for Traumatic Stress Studies. 1993; San Antonio, TX

16. Bliese PD, Wright KM, Adler AB, Cabrera O, Castro CA, Hoge CW. Validating the primary care posttraumatic stress disorder screen and the posttraumatic stress disorder checklist with soldiers returning from combat. J Consult Clin Psychol. 2008;76(2):272-81.

17. Skopp NA, Swanson R, Luxton DD, et al. An examination of the diagnostic efficiency of post-deployment mental health screens. $\mathrm{J}$ Clin Psychol. 2012;68(12): 1253-65.

18. Defense Technical Information Center. Department of Defense instruction: mental health assessments for service members deploiyed in connection with a contingency operation. 2013.
Available at http://www.dtic.mil/whs/directives/corres/pdf/ 649012p/pdf. Accessed September 8, 2015.

19. Department of Veterans Affairs. Vista clinical reminder user manual 2007. Available at http://www.va.gov/vdl/documents/clinical/CPRS_ Clinical_Reminders_2_6_um.pdf. Accessed September 8, 2015.

20. American Psychiatric Association. Diagnostic and statistical manual of mental disorders-5th edition (DSM-5). Arlington: American Psychiatric Association; 2013

21. Friedman MJ, Resick PA, Bryant RA, Strain J, Horowitz M, Spiegel D. Classification of trauma and stressor-related disorders in DSM-5. Depress Anxiety. 2011;28(9):737-49.

22. Friedman MJ, Resick PA, Bryant RA, Brewin CR. Considering PTSD for DSM-5. Depress Anxiety. 2011;28(9):750-69.

23. King DW, Leskin GA, King LA, Weathers FW. Confirmatory factor analysis of the clinician-administered PTSD scale: evidence for the dimensionality of posttraumatic stress disorder. Psychol Assess. 1998; 10(2):90-6.

24. Keane TM, Rubin A, Lachowicz M, et al. Temporal stability of DSM-5 posttraumatic stress disorder criteria in a problem-drinking sample. Psychol Assess. 2014;26(4):1138-45.

25. DeWalt DA, Rothrock N, Yount S, Stone AA. Evaluation of item candidates: the PROMIS qualitative item review. Med Care. 2007;45(5 Suppl 1):S12-21.

26. Prins A, Jenkins-Guarnieri M, Smolenski D, et al. Revising the PCPTSD Screen for DSM-5. Paper presented at the annual meeting of the International Society of Traumatic Stress Studies. 2014; Miami, FL.

27. Cicchetti DV. Guidelines, criteria, and rules of thumb for evaluating normed and standardized assessment instruments in psychology. Psychol Assess. 1994;6(4):284-90

28. Tiet QQ, Leyva YE, Moos RH, Frayne SM, Osterberg L, Smith B. Screen of drug use: diagnostic accuracy of a new brief tool for primary care. JAMA Intern Med. 2015;175(8):1371-7.

29. Sheehan DV, Lecrubier $\mathbf{Y}$, Sheehan $\mathbf{K H}$, et al. The Mini-International Neuropsychiatric Interview (MINI): the development and validation of a structured diagnostic psychiatric interview for DSM-IV and ICD-10. J Clin Psychiatry. 1998; 59(Suppl 20):22-33.

30. Sheehan DV, Lecrubier Y, Sheehan KH, et al. The validity of the MiniInternational Neuropsychiatric Interview (MINI) according to the SCID-P and its reliability. Eur Psychiatry, 1997; 12(5):232-41.

31. Lecrubier, $\mathbf{Y}$, Sheehan, DV, Weiller, E, et al. The Mini-International Neuropsychiatric Interview (MINI): reliability and validity according to the CIDI. Eur Psychiatry. 1997; 12(5):224-31.

32. Pinninti, N, Madison, H, Musser, E, Rissmiller, D. MINI Internationa Neuropsychiatric Schedule: clinical utility and patient acceptance. Eur Psychiatry. 2003; 18(7):361-64.

33. Kraemer HC. Evaluating medical tests: objective and quantitative guidelines. Newbury Park: Sage publications; 1992.

34. Morina N, Ehring T, Priebe S. Diagnostic utility of the impact of event scale-revised in two samples of survivors of war. PLoS One. 2013:8(12):e83916.

35. Altman DG. Practical statistics for medical research. CRC Press; 1990

36. Mackinnon A. A spreadsheet for the calculation of comprehensive statistics for the assessment of diagnostic tests and inter-rater agreement. Comput Biol Med. 2000;30(3):127-34.

37. Kroenke K, Spitzer RL, Williams JB. The PHQ-9: validity of a brief depression severity measure. J Gen Intern Med. 2001;16(9):606-13.

38. Hoge CW, Riviere LA, Wilk JE, Herrell RK, Weathers FW. The prevalence of post-traumatic stress disorder (PTSD) in US combat soldiers: a head-tohead comparison of DSM-5 versus DSM-IV-TR symptom criteria with the PTSD checklist. Lancet Psychiatry. 2014; 1(4):269-77.

39. Gros DF, Frueh BC, Magruder KM. Prevalence and features of panic disorder and comparison to posttraumatic stress disorder in VA primary care. Gen Hosp Psychiatry. 2011;33(5):482-8.

40. Kimerling R, Ouimette P, Prins A, et al. BRIEF REPORT: utility of a short screening scale for DSM-IV PTSD in primary care. J Gen Intern Med. 2006;21(1):65-7.

41. Bomyea J, Lang AJ, Golinelli D, et al. Trauma exposure in anxious primary care patients. J Psychopathol Behav Assess. 2013;35(2):254-63.

42. Weathers, FW, Blake, DD, Schnurr, PP, Kaloupek, DG, Marx, BP, Keane,TM. The Clinician-Administered PTSD Scale for DSM-5 (CAPS-5). US Department of Veterans Affairs, National Center for PTSD. 2013. Interview available from the National Center for PTSD at www.ptsd.va.gov.

43. McDonald SD, Calhoun PS. The diagnostic accuracy of the PTSD checklist: a critical review. Clin Psychol Rev. 2010;30(8):976-87. 